\title{
Dynamics of spontaneous emission in a single-end photonic waveguide
}

\author{
Tommaso Tufarelli, ${ }^{1}$ Francesco Ciccarello, ${ }^{2,3}$ and M. S. Kim ${ }^{1}$ \\ ${ }^{1}$ QOLS, Blackett Laboratory, Imperial College London, SW7 2BW, United Kingdom \\ ${ }^{2}$ Scuola Normale Superiore, Piazza dei Cavalieri, 7, I-56126 Pisa, Italy \\ ${ }^{3}$ NEST, Istituto Nanoscienze-CNR and Dipartimento di Fisica, Università degli Studi di Palermo, via Archirafi 36, I-90123 Palermo, Italy
}

(Received 14 August 2012; published 18 January 2013)

\begin{abstract}
We investigate the spontaneous emission of a two-level system, e.g., an atom or atomlike object, coupled to a single-end, i.e., a semi-infinite, one-dimensional photonic waveguide such that one end behaves as a perfect mirror while light can pass through the opposite end with no backreflection. Through a quantum microscopic model we show that such geometry can cause nonexponential and long-lived atomic decay. Under suitable conditions, a bound atom-photon stationary state appears in the atom-mirror interspace so as to trap a considerable amount of initial atomic excitation. Yet this can be released by applying an atomic frequency shift, causing a revival of photon emission. The resilience of such effects to typical detrimental factors is analyzed.
\end{abstract}

DOI: 10.1103/PhysRevA.87.013820 PACS number(s): 42.50.Ct, 42.50.-p, 42.50.Nn, 52.25.Os

\section{INTRODUCTION}

A major, if not distinctive, line in quantum electrodynamics (QED) is to study how geometric constraints affect the interaction between atomic systems and the electromagnetic (EM) field. On the one hand, this can bring a deeper insight into the related physics. On the other hand, phenomena that spontaneously do not occur in nature can become observable this way. Spontaneous emission (SE) is an elementary process in QED. One normally associates this with an exponential decay of a quantum emitter $(\mathrm{QE})$ to its ground state accompanied by an irreversible release of energy to the EM vacuum (we will often use the term "atom" to refer to the $\mathrm{QE}$, even though this need not be, necessarily, an actual atom). However, free-space SE can be significantly affected - even in its qualitative features-by introducing geometric constraints, forcing the EM field within a certain region of space [1-3] or a lattice structure (see, e.g., [4]). Cavity QED has embodied for a long time the traditional test bed for investigating such effects. Nowadays, growing technologic capabilities to effectively confine the EM field within less than three dimensions and making it interact with a small number of atoms are opening the door to yet unexplored areas of QED. In particular, a variety of experimental implementations of one-dimensional (1D) photonic waveguides coupled to few-level systems have been developed. These include photonic-crystal waveguides with defect cavities [5], optical or hollow-core fibers interacting with atoms [6], microwave transmission lines coupled to superconducting qubits [7], semiconducting (diamond) nanowires with embedded QDs (nitrogen vacancies) [8-10], or plasmonic waveguides coupled to QDs or nitrogen vacancies [11] (see Ref. [12] for a more comprehensive list). Interestingly, even free-space setups employing tightly focused photons have the potential to embody effective 1D waveguides [13].

In most cases, the number (even at the level of a single unity) and positioning of such atomlike objects can be accurately controlled. Besides major applicative concerns to study such 1D systems (e.g., some of them can work as highly efficient single-photon sources), these developments are fostering a renewed interest in their fundamental quantum optical properties. Peculiar effects can take place, such as giant Lamb shifts [14] or the ability of an atom to perfectly reflect back an impinging resonant photon due to the destructive interference between spontaneous and stimulated emission [15]. The latter effect is at the heart of attractive applications such as single-photon transistors [16] and atomic light switches [17].

To capture certain features of their physical behavior, it often suffices to model 1D photonic waveguides as endless. In reality, of course, one such structure is terminated on both sides, each end typically lying at the junction between the waveguide itself and a solid state or air medium. Therefore light impinging on either end always undergoes some partial backreflection owing to refractive-index mismatch. Yet, mostly prompted by the wish to realize efficient single-photon sources, the latest technology is now attaining the fabrication of single-end, quasi-1D structures. For instance, this can be achieved by tapering the waveguide toward one end so as to make this almost transparent, while the opposite end is joined to an opaque medium $[9,10]$. The system thus behaves as being semi-infinite. Equivalently, it can be regarded as an infinite waveguide with a perfect mirror (embodied by the opaque end). Given this state of the art, a thorough knowledge of the emission process of an atom in such a configuration is topical. While the analogous problem in three-dimensional (3D) space has been studied extensively [2], first insight into the SE of a $\mathrm{QE}$ in a semi-infinite $1 \mathrm{D}$ waveguide has been acquired only recently through semiclassical $[18,19]$ and quantum models [20]. Unlike the two-dimensional (2D) or 3D cases, the peculiarity of this $1 \mathrm{D}$ setup is that the entire amount of radiation emitted by the atom and backreflected by the mirror is constrained to return to the emitter, and hence has a significant chance to reinteract with it . As is typical in such circumstances, due to multiple reflections, the atom-mirror optical path length becomes crucial and resonances are introduced in the system. This is witnessed by very recent studies (although not focusing on SE), where the waveguide termination was shown to drastically benefit microwave-single-photon detection [21], atomic inversion schemes [22], and processing of quantum information encoded in QEs [23] and photons [24].

In Ref. [20], through a stationary approach suited to atomphoton 1D scattering [15] it was shown that quasibound states can emerge in a single-end waveguide coupled to an atom. 
Indeed, it is known that an atom can behave as a perfect mirror itself $[13,15,17]$; hence effective cavities with atomic mirrors can be formed [25].

Here we study the SE of a two-level system coupled to a semi-infinite waveguide through the analysis of a fully quantum model and a purely dynamical approach. We find that nonexponential emission with long time tails occurs in general. This can feature photon-reabsorption signatures and even excitation trapping, where the latter means that full atomic decay to the ground state is inhibited due to the emergence of an atom-photon bound state. We explain such effects in detail and illustrate the corresponding output light dynamics thanks to a nonperturbative analysis of the system's time evolution, resulting in a closed delay differential equation governing the entire SE process.

The present paper is structured as follows. In Sec. II we introduce our model and explain the method we used to tackle the SE dynamics. Some assumptions that we make are justified. In Sec. III, after working out the delay differential equation governing the atomic excitation time evolution, some typical examples of the entailed dynamics are shown. In particular, we illustrate the inhibition of a full atomic decay to the ground state. In Sec. IV this peculiar effect is demonstrated by working out analytically the excitation amplitude at large times. We also provide a physical explanation by showing that, correspondingly, within the mirror-atom interspace a bound state is formed whose overlap with the initial state matches the asymptotic excitation amplitude. In Sec. V we illustrate the dynamics of the output light exiting the waveguide and show an interesting method to induce an emission revival corresponding to a full release of the trapped excitation. In Sec. VI we analyze how resilient these phenomena are to typical detrimental effects occurring in this type of setups. In Sec. VII we finally draw our conclusions. The work ends with three appendixes, where some technical details are supplied.

\section{MODEL AND APPROACH}

We consider a 1D semi-infinite waveguide along the $x$ axis, whose only termination lies at $x=0$. The waveguide is coupled at $x=x_{0}$ to a two-level atom, whose ground and excited states $|g\rangle$ and $|e\rangle$ have a frequency splitting $\omega_{0}$. Thus $x_{0}$ is the distance between the atom and the waveguide end, the latter behaving as a perfect mirror. We sketch the entire setup in Fig. 1. The waveguide supports a continuum of electromagnetic modes, each with associated wave vector $k$, frequency $\omega_{k}$, and annihilation (creation) operator $\hat{a}_{k}\left(\hat{a}_{k}^{\dagger}\right)$, obeying the bosonic commutation rule $\left[\hat{a}_{k}, \hat{a}_{k^{\prime}}^{\dagger}\right]=\delta\left(k-k^{\prime}\right)$. In the case of an infinite waveguide, for each $k>0$ two orthogonal standing modes are possible with spatial profiles $\propto \cos (k x)$ and $\propto \sin (k x)$, respectively. In our case, given that the waveguide terminates at $x=0$, only the sinelike modes are to be accounted for. Thereby, the atom is dipole coupled to mode $k$ with strength $g_{k} \propto \sin \left(k x_{0}\right)$. By neglecting counter-rotating terms, the Hamiltonian reads

$$
\hat{H}=\omega_{0}|e\rangle\langle e|+\int_{0}^{k_{c}} d k \omega_{k} \hat{a}_{k}^{\dagger} \hat{a}_{k}+\int_{0}^{k_{c}} d k\left(g_{k} \hat{\sigma}_{+} \hat{a}_{k}+\text { H.c. }\right)
$$

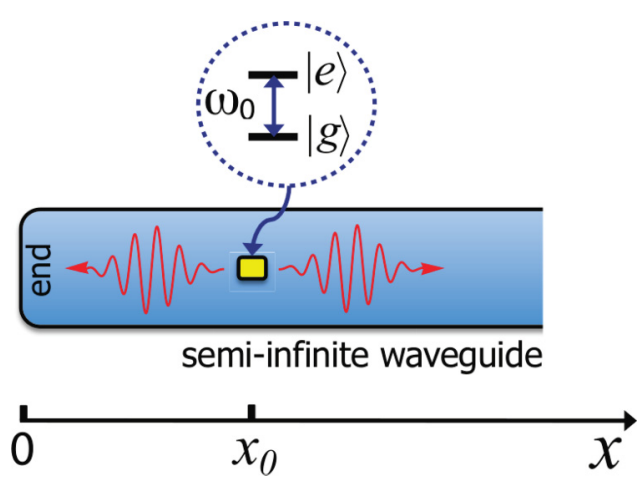

FIG. 1. (Color online) Setup. A semi-infinite waveguide, whose end lies at $x=0$, coupled to a two-level system at $x=x_{0}$.

where $\hat{\sigma}_{+}=\hat{\sigma}_{-}^{\dagger}=|e\rangle\langle g|$ and $k_{c}$ stands for a cutoff wave vector depending on the specific waveguide. The total number of excitations is conserved since $\left[\hat{H},|e\rangle\langle e|+\int d k \hat{a}_{k}^{\dagger} \hat{a}_{k}\right]=0$. As we will focus on the atomic SE, the dynamics occurs entirely within the one-excitation sector of the Hilbert space. Thus at time $t$ the wave function is of the form

$$
|\Psi(t)\rangle=\varepsilon(t)|e\rangle|0\rangle+|g\rangle \int d k \varphi(k, t) a_{k}^{\dagger}|0\rangle,
$$

where $|0\rangle$ is the field vacuum state, $\varepsilon(t)$ is the atomic excitation probability amplitude, and $\varphi(k, t)$ is the field amplitude in the $k$ space (the normalization condition $|\varepsilon(t)|^{2}+\int d k|\varphi(k, t)|^{2}=$ 1 holds). Using Eqs. (1) and (2) and the bosonic commutation rules, the time-dependent Schrödinger equation $\partial_{t}|\Psi\rangle=$ $-i \hat{H}|\Psi\rangle$ yields the coupled differential equations:

$$
\begin{gathered}
\dot{\varepsilon}(t)=-i \omega_{0} \varepsilon(t)-i \int_{0}^{k_{c}} d k g_{k} \varphi(k, t), \\
\partial_{t} \varphi(k, t)=-i \omega_{k} \varphi(k, t)-i g_{k}^{*} \varepsilon(t) .
\end{gathered}
$$

In line with standard approaches for tackling similar systems $[12,15]$, we shall make two main assumptions. First, the photon dispersion relation can be linearized around the atomic frequency as $\omega_{k} \simeq \omega_{0}+v\left(k-k_{0}\right)$, where $v=d \omega /\left.d k\right|_{k=k_{0}}$ is the photon group velocity and $k_{0}$ is such that $\omega_{\kappa_{0}}=\omega_{0}$. Moreover, to simplify our calculations we approximate the integral bounds as $\int_{0}^{k_{c}} d k \rightarrow \int_{-\infty}^{\infty} d k$. These approximations, including the exclusion of the counter-rotating terms mentioned earlier, are valid because we will focus on processes where only a narrow range of wave vectors around $k=k_{0}$ is involved. Hence, wave vectors which are far from $k_{0}$ (including $k<0$ and $k>k_{c}$ that are unphysical) have negligible effect. In the following, we will set $g_{k}=\sqrt{\Gamma v / \pi} \sin k x_{0}$, where $\Gamma$ is the atomic SE rate if the waveguide were infinite (no mirror). This assumption will be justified a posteriori shortly.

\section{SPONTANEOUS EMISSION DYNAMICS}

Next, we study the system's dynamics when the atom and field are initially in $|e\rangle$ and $|0\rangle$, respectively. The initial conditions thus read $\varepsilon(0)=1$ and $\varphi(k, 0)=0$ for any $k$. We start by removing the central frequency $\omega_{0}$ from Eqs. (3) and (4), via the transformation $\varepsilon(t) \rightarrow \varepsilon(t) \mathrm{e}^{-i \omega_{0} t}, \varphi(k, t) \rightarrow \varphi(k, t) \mathrm{e}^{-i \omega_{0} t}$. Equation (4) is thus integrated in terms of the function $\varepsilon(t)$ as $\varphi(k, t)=-i \sqrt{\Gamma v / \pi} \sin k x_{0} \int_{0}^{t} d s e^{i v\left(k-k_{0}\right)(s-t)} \varepsilon(s)$. Replacing 
this into Eq. (3) gives

$$
\dot{\varepsilon}(t)=-\frac{\Gamma v}{\pi} \int_{0}^{t} d s \varepsilon(s) e^{-i v k_{0}(s-t)} \int d k \sin ^{2}\left(k x_{0}\right) e^{i v k(s-t)} .
$$

The integral over $k$ is easily calculated as a linear combination of $\delta\left(s-t \pm t_{d}\right)$ and $\delta(s-t)$, where $t_{d}=2 x_{0} / v$ is the time taken by a photon to travel from the atom to the waveguide end and back (see Fig. 1). Once this is used to carry out the integration over $s$, we end up with a delay differential equation (DDE) for $\varepsilon(t)$ with associated time delay $t_{d}$ :

$$
\dot{\varepsilon}(t)=-\frac{\Gamma}{2} \varepsilon(t)+\frac{\Gamma}{2} e^{i \phi} \varepsilon\left(t-t_{d}\right) \theta\left(t-t_{d}\right),
$$

where $\theta(t)$ is the Heaviside step function while the phase $\phi=$ $2 k_{0} x_{0}$ is the optical length of twice the atom-mirror path (see Fig. 1). DDEs typically occur in problems where retardation effects are relevant, such as in the case of two distant QEs in free space [26] and a single emitter embedded in a dielectric nanosphere [27]. A similar equation was recently obtained in Ref. [21] by working in real space. The first term on the righthand side of Eq. (6) describes a standard damping at a rate $\Gamma$. The second term, instead, indicates that atomic reabsorption of the emitted photon can occur at times $t \geqslant t_{d}$. At earlier times, such a reabsorption term is null since the photon has not yet performed a round trip between the atom and the mirror. Also, it vanishes in the limit $t_{d} \rightarrow \infty$, since the waveguide then effectively becomes infinite and, as expected, the atom undergoes standard, namely, fully irreversible $\mathrm{SE}$ at a rate $\Gamma$ according to $|\varepsilon(t)|^{2}=e^{-\Gamma t}$. This justifies our parametrization of the coefficients $g_{k}$, introduced at the end of the previous section.

Equation (6) can be solved iteratively by partitioning the time axis into intervals of length $t_{d}$. By proceeding similarly to Ref. [26] we obtain its explicit solution as

$$
\varepsilon(t)=e^{-\frac{\Gamma}{2} t} \sum_{n} \frac{1}{n !}\left(\frac{\Gamma}{2} e^{i \phi+\frac{\Gamma}{2} t_{d}}\right)^{n}\left(t-n t_{d}\right)^{n} \theta\left(t-n t_{d}\right),
$$

where the effect of multiple reflection and reabsorption events is witnessed by the presence of the Heaviside step functions (they add a new contribution to the sum at the end of each photon round trip). In Fig. 2 we plot the time evolution of the atom excitation probability $P_{e}(t)=|\varepsilon(t)|^{2}$ for different values of $\phi$ for $\Gamma t_{d}=2$ (a) and $\Gamma t_{d}=0.1(\mathrm{~b})$. In either case, the expected (a)

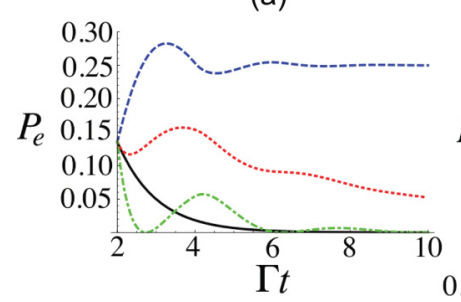

(b)

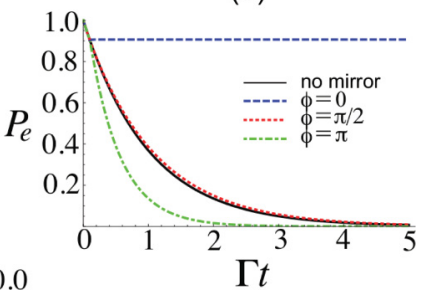

FIG. 2. (Color online) Atomic excitation probability $P_{e}(t)=$ $|\varepsilon(t)|^{2}$ vs time (in units of $1 / \Gamma$ ) in the cases $\Gamma t_{d}=2$ (a) and $\Gamma t_{d}=0.1$ (b) and for $t_{d}=\infty$ (i.e., no mirror; solid black line), $\phi=2 n \pi$ (blue dashed line), $\phi=\pi / 2+2 n \pi$ (red dotted), and $\phi=(2 n+1) \pi$ (green dash-dotted). In (a), only the range $t \geqslant t_{d}$ is shown; at earlier times the behavior does not depend on $\phi$ and is the continuation of the black solid line. purely exponential decay occurring with an infinite waveguide (i.e., the no-mirror case) is displayed for comparison. Such behavior clearly takes place even in the present setup as long as $t<t_{d}$ (independently of $\phi$ and $t_{d}$ ). As soon as $t \geqslant t_{d}$, however, the presence of the mirror starts affecting the atom in a way that the dynamics is now strongly dependent on $\phi$ and $t_{d}$. For $\Gamma t_{d}$ of the order of 1, such as in Fig. 2(a), the behavior of the atomic population can deviate sensibly from an exponential decay: it exhibits one or more peaks of partial atomic reexcitation and, eventually, a monotonic decay. The phase $\phi$ affects both the positions of such re-excitation peaks and the long-time behavior of $P_{e}(t)$ [see Fig. 2(a)]. When instead $\Gamma t_{d} \ll 1$, such as in Fig. 2(b), $P_{e}\left(t \geqslant t_{d}\right)$ drops monotonically with the phase $\phi$, simply affecting the atom's average lifetime. Indeed, in such regimes the solution to Eq. (6) can be approximated as (see Appendix A)

$$
\varepsilon(t) \simeq e^{-\frac{\Gamma}{2} t} \theta\left(t_{d}-t\right)+e^{-\frac{\Gamma t_{d}}{2}}\left(\frac{1+e^{i \phi} \frac{\Gamma t_{d}}{2}}{1+\frac{\Gamma t_{d}}{2}}\right)^{\frac{t-t_{d}}{t_{d}}} \theta\left(t-t_{d}\right),
$$

up to an irrelevant phase factor. The corresponding $|\varepsilon(t)|^{2}$ decays monotonically since $\left|1+e^{i \phi \frac{\Gamma t_{d}}{2}}\right| \leqslant\left|1+\frac{\Gamma t_{d}}{2}\right|$.

\section{ATOM-PHOTON BOUND STATE}

An interesting feature emerges for $\phi=0$. Figure 2 indeed shows that, regardless of $\Gamma t_{d}$, such optical path length inhibits a full excitation decay of the atom on the considered time scales. Indeed, it can be shown that the atom holds a significant amount of excitation even in the limit $t \rightarrow \infty$. To show this, we take the Laplace transform (LT) of Eq. (6) and solve the resulting algebraic equation. This yields

$$
\tilde{\varepsilon}(s)=\frac{1}{s+\frac{\Gamma}{2}\left(1-e^{i \phi-s t_{d}}\right)},
$$

where $\tilde{\varepsilon}(s)$ is the LT of $\varepsilon(t)$. Using the final value theorem, we find the long-time limit [28]

$$
\begin{aligned}
\varepsilon(t \rightarrow \infty) & =\lim _{s \rightarrow 0}[s \tilde{\varepsilon}(s)] \\
& =\left\{\begin{array}{ll}
\left(1+\frac{\Gamma t_{d}}{2}\right)^{-1} & \text { for } \phi=2 n \pi \\
0 & \text { for } \phi \neq 2 n \pi
\end{array} .\right.
\end{aligned}
$$

In the former case note that, in particular, the asymptotic excitation increases when $t_{d}$ is reduced, witnessing the crucial presence of the mirror. The lower $\Gamma$ the more significant is the increase, i.e., the less uncertain is the atomic emitted light wavelength, the more pronounced is the effect, suggesting an interference-like mechanism behind the phenomenon. Such inhibition of spontaneous emission can be interpreted as due to a destructive interference between the different paths that the emitted photon can take to exit the waveguide, or equivalently, between the probability amplitudes of emitting the photon at two different times. It is indeed the signature of a metastable bound state established between the atom and the photonic environment. The emergence of atom-photon bound states has been demonstrated in other different scenarios such as gapped photonic crystals [4] and superohmic baths [29]. 
If existent, an atom-photon bound state $\left|\Psi_{b}\right\rangle=\varepsilon_{b}|e 0\rangle+$ $\int d k \varphi_{b}(k)|g\rangle \hat{a}_{k}^{\dagger}|0\rangle$ must fulfill the normalization condition $\left\langle\Psi_{b} \mid \Psi_{b}\right\rangle=1$ and the time-independent Schrödinger equation $\hat{H}\left|\Psi_{b}\right\rangle=E\left|\Psi_{b}\right\rangle$. With the replacement $\partial_{t} \rightarrow-i E$ in Eqs. (3) and (4), once the rescaled energy parameter $q=\left(E-\omega_{0}\right) / v$ is introduced, we end up with the following equations for the atomic and field amplitudes:

$$
\begin{gathered}
q \varepsilon_{b}=\sqrt{\Gamma /(v \pi)} \int d k \sin \left(k x_{0}\right) \varphi_{b}(k), \\
{\left[q-\left(k-k_{0}\right)\right] \varphi_{b}(k)=\sqrt{\Gamma /(v \pi)} \sin \left(k x_{0}\right) \varepsilon_{b} .}
\end{gathered}
$$

Solving Eq. (12) for $\varphi_{b}(k)$, we obtain that the squared norm of the bound state is given by $\left\langle\Psi_{b} \mid \Psi_{b}\right\rangle=[1+$ $\left.\Gamma /(\pi v) \int d k \sin ^{2}\left(k x_{0}\right) /\left(k-k_{0}-q\right)^{2}\right]\left|\varepsilon_{b}\right|^{2}$. Calculating the integral over $k$ through standard contour-integration methods and imposing the normalization condition yields

$$
\left|\varepsilon_{b}\right|^{2}=\left(1+\frac{\Gamma t_{d}}{2} \cos \left[2\left(k_{0}+q\right) x_{0}\right]\right)^{-1} .
$$

We now replace $\varphi_{b}(k)$ as given by Eq. (12) in Eq. (11), making use again of contour integration, so as to end up with a consistency equation for the rescaled energy parameter:

$$
q=-\frac{\Gamma}{2 v} \sin \left[2\left(k_{0}+q\right) x_{0}\right]
$$

It is now easy to show that $q=0$, i.e., $E=\omega_{0}$. We start by noticing that we can find a value of $k$ that makes the left-hand side of Eq. (12) vanish, namely, $k=k_{0}+q$. This then entails $\sin \left[\left(k_{0}+q\right) x_{0}\right]=0$, that is, $\left(k_{0}+q\right) x_{0}=n \pi$ ( $n$ is an integer $)$, implying that also the right-hand side of Eq. (14) vanishes, so that $q=0$ and $\phi=2 k_{0} x_{0}=2 n \pi$. In conclusion, for $\phi=0$ $(\bmod 2 \pi)$ a bound state $\left|\Psi_{b}\right\rangle$ having energy $E=\omega_{0}$ arises, which significantly overlaps the excited state (the overlap being $\left\langle e 0 \mid \Psi_{b}\right\rangle=\varepsilon_{b}$ ). This explains why full atomic decay to the ground state is inhibited: the projection of the excited state onto the bound state does not couple to the traveling photons, so that, at long times, $|e 0\rangle \rightarrow\left|\Psi_{b}\right\rangle\left\langle\Psi_{b} \mid e 0\right\rangle+$ (field terms) and $\varepsilon(t \rightarrow \infty)=\left|\varepsilon_{b}\right|^{2}$. As is easily checked [30], the amount of atomic excitation corresponding to this overlap remains confined within the interval $0 \leqslant x \leqslant x_{0}$, shared between the atom and photonic field.

This is interpreted as follows. As mentioned, an atom on resonance with a traveling photon behaves as a perfect mirror $[15,17]$. Thus, just like in a standard Fabry-Perot interferometer, one expects a field standing wave to arise within $0 \leqslant x \leqslant x_{0}$ when the emitted wavelength matches $x_{0}$. This agrees with the findings in Ref. [20], which were however derived in the strong coupling limit. Our $k$-space approach thus allows proof that a bound state is indeed created and works out explicitly its exact form.

\section{OUTPUT FIELD DYNAMICS}

So far we have focused on the atomic excitation dynamics. A natural way to experimentally test this is to measure the light emitted through the free, i.e., nonreflective, end of the waveguide. It is then important to study the entailed dynamics of such output light. The real-space field annihilation operator
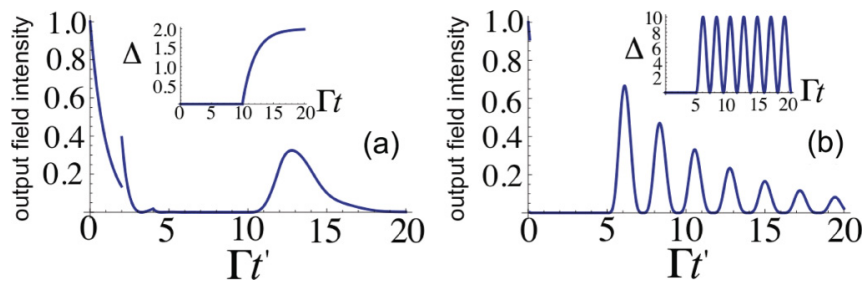

FIG. 3. (Color online) Output field intensity vs $t^{\prime}=t-d / v$ in arbitrary units for $\phi=2 n \pi$ under an applied frequency shift $\Delta(t)$ (this is plotted in the insets in units of $\Gamma$ ). (a) $\Gamma t_{d}=2$ and a steplike $\Delta(t)$. (b) $\Gamma t_{d}=0.1$ and a sinusoidal $\Delta(t)$. In either case, a revival of the photon emission occurs when the frequency shift is switched on. Note that, in line with Eq. (17), at $t=t_{d}$ the intensity exhibits a discontinuity [particularly visible in (a)].

at position $x>0$ can be expressed as

$$
\hat{C}(x)=\sqrt{\frac{2}{\pi}} \int d k \hat{a}_{k} \sin k x,
$$

where the prefactor stems from the normalization constraint $\int_{0}^{\infty} d x \hat{C}^{\dagger}(x) \hat{C}(x)=\int_{0}^{\infty} d k \hat{a}_{k}^{\dagger} \hat{a}_{k}$. Once applied to the state in Eq. (2), this yields $\hat{C}(x)|\Psi(t)\rangle=\psi(x, t)|g\rangle|0\rangle$, where

$$
\psi(x, t)=\sqrt{\frac{2}{\pi}} \int d k \varphi(k, t) \sin k x
$$

can be interpreted as the real-space field amplitude. The square modulus of $\psi(x, t)$ can be measured via the local photon density, which is $\propto\left\langle\Psi(t)\left|\hat{C}^{\dagger}(x) \hat{C}(x)\right| \Psi(t)\right\rangle=|\psi(x, t)|^{2}$. We assume that a photon detector lies at position $\bar{x}=x_{0}+$ $d$, where $d>0$ is the atom-detector distance. Hence (see Appendix B)

$$
\begin{aligned}
\psi(\bar{x}, t) & =\sqrt{\frac{\Gamma}{2 v}} e^{i k_{0} d}\left[\varepsilon\left(t^{\prime}\right) \theta\left(t^{\prime}\right)-e^{i \phi} \varepsilon\left(t^{\prime}-t_{d}\right) \theta\left(t^{\prime}-t_{d}\right)\right] \\
& =\sqrt{\frac{2}{\Gamma v}} e^{i k_{0} d} \dot{\varepsilon}\left(t^{\prime}\right) \theta\left(t^{\prime}\right),
\end{aligned}
$$

where $t^{\prime}=t-d / v$, and the last equality follows from Eq. (6). Equation (18) shows that the time evolution of the atomic excitation (once the time delay $d / v$ is accounted for) can in fact be obtained by integrating the output field amplitude over time. The latter can be retrieved from the field intensity in the special cases $\phi=0, \pi$, in which the phase of $\varepsilon$ is constant, while in general homodyne techniques will be required. Clearly, for $\phi=2 n \pi$, which ensures the formation of the atom-photon bound state (see previous section), the atom cannot fully decay to the ground state and thus less than one photon exits the waveguide on average. An interesting simple method exists, though, to force the trapped excitation to be released. At a time long enough that the unbound excitation has left the waveguide, an atomic frequency shift $\Delta$ is applied (this is routinely implemented through local fields). As this changes the atomic frequency $\omega_{0}$ and thereby the corresponding $k_{0}$, $\phi$ is modified as well. This suppresses the bound state and necessarily compels the trapped excitation to leak out as light. In Fig. 3, we model the frequency shift switch as a smooth time function $\Delta(t)$ [in a way that in Eq. (1) $\omega_{0} \rightarrow \omega_{0}+\Delta(t)$, while in Eqs. (6) and (18) $\dot{\varepsilon}(t) \rightarrow \dot{\varepsilon}(t)+i \Delta(t) \varepsilon(t)]$ and plot the resulting numerically computed output field intensity against 
time. Clearly, as soon as $\Delta(t) \neq 0$ a spontaneous emission revival takes place, witnessing that release of the bound-state excitation has been triggered. For $\Gamma t_{d} \ll 1$ [Fig. 3(b)], the initial emission (when $\Delta$ is still zero) is almost negligible because in such regimes most of the energy is trapped within the bound state [cf. Eq. (10) and Fig. 2(b) for $\phi=0$ ]. Interestingly, in such a case the system responds quickly to applied frequency shifts, which can be understood as follows. We start by observing that a nonzero value of $\Delta$ is equivalent to an appropriate phase shift $\phi \rightarrow \phi+\delta \phi$. When the phase is shifted from the value $2 n \pi$, the bound state is suppressed and the photonic emission revived. From the approximate solution of Eq. (8), one can see that after a transient $\simeq t_{d}$ the emission rate stabilizes to a fixed value. If later the phase is restored to the bound-state value, Eq. (8) again indicates that the system ceases to emit after a further transient time $\simeq t_{d}$, occurring in a small unwanted excitation loss $\sim \Gamma t_{d}$. Hence, when $\Gamma t_{d} \ll 1$ these transients have a minor effect so as to allow for a satisfactory degree of control over the atomic emission.

As a result, the shape of $\Delta(t)$ is closely reflected in the temporal profile of the light intensity, as shown in Fig. 3(b) in a paradigmatic case. Such effect has the potential to be harnessed to emit single-photon pulses directionally and with controllable temporal profiles, which can be of concern to a variety of fields, especially in connection with quantum information technologies. The method outlined here shares some similarities with earlier cavity QED proposals for the deterministic generation of single photons, which typically require the use of more than two internal atomic levels combined with adiabatic transfer techniques [31]. In contrast, in our setup the existence of a metastable bound state allows for the control of the atomic emission without the need for extra degrees of freedom and through the simple application of a classical field detuning the atom.

\section{RESILIENCE TO DETRIMENTAL EFFECTS}

To assess the experimental observability of the central phenomena presented so far, we have refined the model to account for detrimental factors. In addition to the waveguide modes, we allow for an extra atomic coupling to a reservoir of external nonaccessible modes at a rate $\Gamma_{\text {ext }}$. Also, we assume that the guide is terminated at $x=0$ with a nonideal mirror of reflectivity $R<1$. Moreover, we introduce (inhomogeneous) phase noise on the atom by adding a small white-noise stochastic term to the excited-state frequency as $\omega(t)=\omega_{0}+$ $\eta(t)$. Here, $\eta(t)$ is a Gaussian-distributed random variable such that $\langle\eta(t)\rangle=0$ and $\left\langle\eta(t) \eta\left(t^{\prime}\right)\right\rangle=2 \delta \omega \delta\left(t-t^{\prime}\right)(\langle\cdots\rangle$ stands for the ensemble average), where $\delta \omega$ represents the associated dephasing rate. The corresponding excitation probability $P_{e}(t)=\left\langle|\varepsilon(t)|^{2}\right\rangle$ in such nonideal conditions can be predicted through a semi-analytical procedure (see Appendix C).

The first photon reabsorption peak of $P_{e}(t)$ occurring for $\Gamma t_{d} \sim 1$ [cf. Fig. 2(a)] is rather robust to dissipation into external modes (expected to be the major detrimental factor affecting such a specific feature). As shown in Fig. 4(a), a "shoulder" is still visible even with $\Gamma_{\text {ext }}=\Gamma$ (even higher values ensure that SE significantly departs from the mirrorless case). As for the resilience of the bound-state effects, which are stronger for $\Gamma t_{d} \ll 1$ [cf. Fig. 2(b)], Fig. 4(b) shows that in (a)

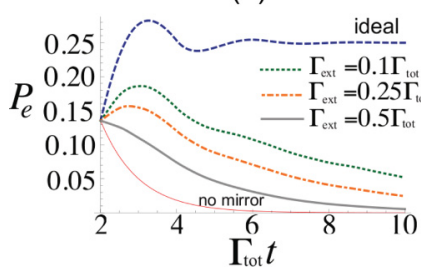

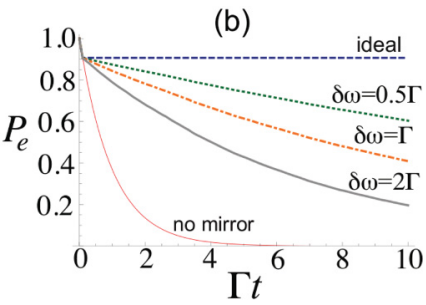

FIG. 4. (Color online) Robustness of $P_{e}(t)=\left\langle|\varepsilon(t)|^{2}\right\rangle$ vs time for $\phi=2 n \pi$. We have studied the dynamics of the excitation amplitude via Eq. (C1), using $\Gamma_{\text {tot }} \equiv \Gamma+\Gamma_{\text {ext }}$, and $r=R+i \sqrt{R(1-R)}$. (a) We set $\Gamma_{\text {tot }} t_{d}=2, R=0.98, \delta \omega=0.25 \Gamma_{\text {tot }}$ and vary the ratio between $\Gamma_{\text {ext }}$ and $\Gamma$, keeping $\Gamma_{\text {tot }}$ fixed. (b) We fix $R=1, \Gamma_{\text {ext }}=0$, $\Gamma t_{d}=0.1$, and vary the dephasing rate $\delta \omega$.

the case of pure dephasing, a significantly long-lived excitation trapping still survives for relatively high $\delta \omega / \Gamma$ ratios.

\section{CONCLUSIONS}

We have investigated the time evolution of spontaneous emission for a two-level system coupled to a semi-infinite 1D photonic waveguide. We have derived an exact delay differential equation for the atomic excitation amplitude. According to this, the atomic excitation undergoes a nonexponential decay which can exhibit oscillations (a signature of partial photon reabsorption) and long time tails. A full decay to the ground state is even inhibited when the emitted wavelength matches the atom-mirror distance, owing to the formation of an atom-photon bound state which we exactly derive. The amount of trapped excitation can be substantial, and it can be released as a photon by applying a frequency shift to the atom, resulting in a light emission revival. We have assessed that such phenomena can be observable even in the presence of substantial detrimental effects such as dissipation into unwanted modes and atomic dephasing. This indicates that an experimental demonstration of the key features investigated here may be not far-fetched. We finally point out that an interesting way to regard our system is to consider the mirror as a means to introduce a feedback mechanism. This ensures that part of the output signal, i.e., the spontaneously emitted light, is re-inserted into the atomic system as input. Significantly, delay differential equations with a similar structure as Eq. (6) occur in quantum optics settings with feedback [32].

\section{ACKNOWLEDGMENTS}

T.T. and M.S.K. acknowledge support from the NPRP 4554-1-084 from Qatar National Research Fund. F.C. acknowledges support from FIRB IDEAS (Project RBID08B3FM). We are grateful to V. Giovannetti, B. Garraway, J. Hwang, D. Dorigoni, M. Tame, M. Reimer, A. Nazir, M. Agio, D. Valente, and C. Benedetti for useful discussions.

\section{APPENDIX A: DERIVATION OF Eq. (8)}

For $t \leqslant t_{d}$, the delay term in Eq. (6) vanishes and thus $\varepsilon(t)=$ $e^{-\Gamma t / 2}$. For $t>t_{d}$ and $\Gamma t_{d} \ll 1$, the time delay $t_{d}$ becomes the shortest time scale in a way that it can be taken as the differential of time. We thus introduce the discrete variable $n$ and, accordingly, define $\varepsilon_{n} \equiv \varepsilon\left(n t_{d}\right)$. Therefore $\dot{\varepsilon} \simeq\left(\varepsilon_{n+1}-\varepsilon_{n}\right) / t_{d}$, 
which once replaced in Eq. (6) gives the recursion identity

$$
\varepsilon_{n+1}=\left(\frac{1+e^{i \phi \frac{\Gamma t_{d}}{2}}}{1+\frac{\Gamma t_{d}}{2}}\right) \varepsilon_{n} .
$$

Using this along with the matching condition at $t=t_{d}$, $\varepsilon_{1}=\varepsilon\left(t_{d}\right)=e^{-\left(\Gamma t_{d} / 2\right)}$, we immediately end up with

$$
\varepsilon_{n}=\left(\frac{1+e^{i \phi} \frac{\Gamma t_{d}}{2}}{1+\frac{\Gamma t_{d}}{2}}\right)^{n-1} e^{-\left(\Gamma t_{d} / 2\right)}
$$

By combining the functions for $t<t_{d}$ and $t>t_{d}$ and reintroducing the continuous time through $n=t / t_{d}$, we find Eq. (8) of the main text.

\section{APPENDIX B: DERIVATION OF Eq. (17)}

We start by recalling that the integration in Eq. (4) of the main text for $\omega_{k}=\omega_{0}+v\left(k-k_{0}\right)$ gives

$$
\varphi(k, t)=-i g_{k} \int_{0}^{t} d s e^{-i v\left(k-k_{0}\right)(t-s)} \varepsilon(s),
$$

where $g_{k}=\sqrt{\Gamma v / \pi} \sin k x_{0}$ and the irrelevant phase factor $e^{-i \omega_{0} t}$ has been removed from both functions $\varphi(k, t)$ and $\varepsilon(t)$. Thus, using that the field amplitude in position space is defined as $\psi(x, t)=\sqrt{2 / \pi} \int d k \varphi(k, t) \sin k x$, we find

$$
\begin{aligned}
\psi(x, t)= & -i \frac{\sqrt{2 \Gamma v}}{\pi} \int_{0}^{t} d s \int d k \sin \left(k x_{0}\right) \\
& \times \sin (k x) e^{-i v\left(k-k_{0}\right)(t-s)} \varepsilon(s) .
\end{aligned}
$$

The integral over $k$ returns a combination of $\delta$ functions, which makes the time integration particularly straightforward. This yields

$$
\begin{aligned}
\psi(x, t) & \\
= & -i \sqrt{\frac{\Gamma}{2 v}}\left[e^{i k_{0}\left(x_{0}-x\right)} \varepsilon\left(t-\frac{x_{0}-x}{v}\right) \theta\left(x_{0}-x\right) \theta\left(v t-x_{0}+x\right)\right. \\
& +e^{i k_{0}\left(x-x_{0}\right)} \varepsilon\left(t-\frac{x-x_{0}}{v}\right) \theta\left(x-x_{0}\right) \theta\left(v t-x+x_{0}\right) \\
& \left.-e^{i k_{0}\left(x+x_{0}\right)} \varepsilon\left(t-\frac{x+x_{0}}{v}\right) \theta\left(x+x_{0}\right) \theta\left(v t-x-x_{0}\right)\right] .
\end{aligned}
$$

In the special case $\bar{x}=x_{0}+d(d>0)$ we have

$$
\psi(\bar{x}, t)=-i \sqrt{\frac{\Gamma}{2 v}} e^{i k_{0} d}\left[\varepsilon\left(t^{\prime}\right) \theta\left(t^{\prime}\right)-e^{i \phi} \varepsilon\left(t^{\prime}-t_{d}\right) \theta\left(t^{\prime}-t_{d}\right)\right],
$$

where $t^{\prime}=t-d / v$ as in the main text. Equation (B4) is equivalent to Eq. (17) of the main text, up to an irrelevant phase factor $-i$.

\section{APPENDIX C: INCLUDING DETRIMENTAL EFFECTS}

Here we briefly explain how we have extended our model so as to include losses and perform the robustness study in Fig. 4 of the main text. For our purposes, it suffices to adopt a heuristic reasoning (a more rigorous analysis yields the same results). The inclusion of a reservoir of external nonaccessible modes is a routine procedure in the literature. It simply amounts to adding a term $-\frac{\Gamma_{\text {ext }}}{2} \varepsilon(t)$ on the right-hand side of Eq. (6), where $\Gamma_{\text {ext }}$ is the decay rate associated to such unwanted modes. The presence of an imperfect mirror with $R<1$ will instead modify the delay term in Eq. (6), since the atom will reinteract only with the portion of light which is reflected. This suggests the substitution $e^{i \phi} \rightarrow r e^{i \phi}$, where $-r$ is the complex probability amplitude for backward reflection off the mirror $\left(|r|^{2}=R\right)$. Solving the 1D scattering problem yields $r=R+i \sqrt{R(1-R)}$. Finally, as mentioned in the main text, we include extra dephasing of the atom by adding a white-noise term to the excited-state frequency as $\omega(t)=\omega_{0}+\eta(t)$, where $\eta(t)$ is a Gaussian-distributed random variable: $\langle\eta(t)\rangle=0$ and $\left\langle\eta(t) \eta\left(t^{\prime}\right)\right\rangle=2 \delta \omega \delta\left(t-t^{\prime}\right)$, where $\delta \omega$ quantifies the strength of the dephasing $[\langle\cdots\rangle$ stands for the ensemble average]. In conclusion, Eq. (6) of the main text is modified as follows:

$$
\begin{aligned}
\dot{\varepsilon}(t)+i \eta(t) \varepsilon(t)= & -\frac{\Gamma+\Gamma_{\mathrm{ext}}}{2} \varepsilon(t) \\
& +r \frac{\Gamma}{2} e^{i \phi} \varepsilon\left(t-t_{d}\right) \theta\left(t-t_{d}\right) .
\end{aligned}
$$

For completeness, we also mention that a similar analysis can be carried out on the output field amplitude, which modifies Eq. (17) of the main text as

$$
\psi(\bar{x}, t)=\sqrt{\frac{\Gamma}{2 v}} e^{i k_{0} d}\left[\varepsilon\left(t^{\prime}\right) \theta\left(t^{\prime}\right)-r e^{i \phi} \varepsilon\left(t^{\prime}-t_{d}\right) \theta\left(t^{\prime}-t_{d}\right)\right] .
$$

To obtain each line in Fig. 4 of the main text, we have integrated Eq. (C1) numerically for 100 realizations in the presence of simulated white noise, and then averaged over these the resulting probabilities $P_{e}(t)=|\varepsilon(t)|^{2}$.

Finally, let us stress again that Eqs. (C1) and (C2) can be obtained rigorously by modifying the microscopic model given by Eq. (1) of the main text. In particular, one has to include both sine and cosine waves for each wave vector $k$, while the presence of an imperfect mirror at $x=0$ can be modeled by adding an extra term in the Hamiltonian of the form $V \hat{C}^{\dagger}(0) \hat{C}(0)$. Here, $V=v \sqrt{R /(1-R)}$ and $\hat{C}(x)$ is the field annihilation operator in real space as introduced in the main text [now, however, owing to the cosine standing modes $\hat{C}(0) \neq 0]$.
[1] E. M. Purcell, Phys. Rev. 69, 681 (1946).

[2] D. Meschede, Phys. Rep. 211, 201 (1992).

[3] H. Walther, B. T. H. Varcoe, B.-G. Englert, and T. Becker, Rep. Prog. Phys. 69, 1325 (2006); R. Miller, T. E. Northup, K. M. Birnbaum, A. Boca, A. D. Boozer, and H. J. Kimble, J. Phys. B 38, S551 (2005); J. M. Raimond, M. Brune, and S. Haroche, Rev. Mod. Phys. 73, 565 (2001).
[4] S. John and J. Wang, Phys. Rev. Lett. 64, 2418 (1990); S. Bay, P. Lambropoulos, and K. Molmer, ibid. 79, 2654 (1997).

[5] A. Faraon, E. Waks, D. Englund, I. Fushman, and J. Vučković, Appl. Phys. Lett. 90, 073102 (2007).

[6] B. Dayan, A. S. Parkins, Takao Aoki, E. P. Ostby, K. J. Vahala, and H. J. Kimble, Science 319, 1062 (2008); E. Vetsch, D. Reitz, G. Sague, R. Schmidt, S. T. Dawkins, and A. Rauschenbeutel, 
Phys. Rev. Lett. 104, 203603 (2010); M. Bajcsy, S. Hofferberth, V. Balic, T. Peyronel, M. Hafezi, A. S. Zibrov, V. Vuletic, and M. D. Lukin, ibid. 102, 203902 (2009).

[7] A. Wallraff, D. I. Schuster, A. Blais, L. Frunzio, R.-S. Huang, J. Majer, S. Kumar, S. M. Girvin, and R. J. Schoelkopf, Nature (London) 431, 162 (2004); O. Astafiev, A. M. Zagoskin, A. A. Abdumalikov, Jr., Yu. A. Pashkin, T. Yamamoto, K. Inomata, Y. Nakamura, and J. S. Tsai, Science 327, 840 (2010).

[8] M. H. M. van Weert, N. Akopian, U. Perinetti, M. P. van Kouwen, R. E. Algra, M. A. Verheijen, E. P. A. M. Bakkers, L. P. Kouwenhoven, and V. Zwiller, Nano Lett. 9, 1989 (2009); T. M. Babinec, B. J. M. Hausmann, M. Khan, Y. Zhang, J. R. Maze, P. R. Hemmer, and M. Lončar, Nat. Nanotechnol. 5, 195 (2010).

[9] J. Claudon, J. Bleuse, N. S. Malik, M. Bazin, P. Jaffrennou, N. Gregersen, C. Sauvan, P. Lalanne, and J.-M. Gérard, Nat. Photonics 4, 174 (2010); J. Bleuse, J. Claudon, M. Creasey, N. S. Malik, J. M. Gerard, I. Maksymov, J. P. Hugonin, and P. Lalanne, Phys. Rev. Lett. 106, 103601 (2011).

[10] M. E. Reimer, G. Bulgarini, N. Akopian, M. Hocevar, M. B. Bavinck, M. A. Verheijen, E. P. A. M. Bakkers, L. P. Kouwenhoven, and Val Zwiller, Nat. Commun. 3, 737 (2012); G. Bulgarini, M. E. Reimer, T. Zehender, M. Hocevar, E. P. A. M. Bakkers, L. P. Kouwenhoven, and V. Zwiller, Appl. Phys. Lett. 100, 121106 (2012).

[11] A. V. Akimov, A. Mukherjee, C. L. Yu, D. E. Chang, A. S. Zibrov, P. R. Hemmer, H. Park, and M. D. Lukin, Nature (London) 450, 402 (2007); A. Huck, S. Kumar, A. Shakoor, and U. L. Andersen, Phys. Rev. Lett. 106, 096801 (2011).

[12] D. Witthaut and A. S. Sørensen, New J. Phys. 12, 043052 (2010).

[13] G. Zumofen, N. M. Mojarad, V. Sandoghdar, and M. Agio, Phys. Rev. Lett. 101, 180404 (2008); N. Lindlein, R. Maiwald, H. Konermann, M. Sondermann, U. Peschel, and G. Leuchs, Laser Phys. 17, 927 (2007).

[14] P. Horak, P. Domokos, and H. Ritsch, Europhys. Lett. 61, 459 (2003).

[15] J.-T. Shen and S. Fan, Opt. Lett. 30, 2001 (2005); Phys. Rev. Lett. 95, 213001 (2005).

[16] D. E. Chang, A. S. Sørensen, E. A. Demler, and M. D. Lukin, Nat. Phys. 3, 807 (2007).
[17] L. Zhou, Z. R. Gong, Y. X. Liu, C. P. Sun, and F. Nori, Phys. Rev. Lett. 101, 100501 (2008).

[18] I. Friedler, C. Sauvan, J. P. Hugonin, P. Lalanne, J. Claudon, and J. M. Gérard, Opt. Express 17, 2095 (2009).

[19] A. V. Maslov, M. I. Bukanov, and C. Z. Ning, J. Appl. Phys. 99, 024314 (2006).

[20] H. Dong, Z. R. Gong, H. Ian, L. Zhou, and C. P. Sun, Phys. Rev. A 79, 063847 (2009).

[21] B. Peropadre, G. Romero, G. Johansson, C. M. Wilson, E. Solano, and J. J. Garcia-Ripoll, Phys. Rev. A 84, 063834 (2011).

[22] Y. Chen, M. Wubs, J. Mørk, and A. F. Koenderink, New J. Phys. 13, 103010 (2011).

[23] F. Ciccarello, D. E. Browne, L. C. Kwek, H. Schomerus, M. Zarcone, and S. Bose, Phys. Rev. A 85, 050305(R) (2012).

[24] D. Valente, Y. Li, J. P. Poizat, J. M. Gerard, L. C. Kwek, M. F. Santos, and A. Auffeves, New J. Phys. 14, 083029 (2012); Phys. Rev. A 86, 022333 (2012).

[25] L. Zhou, H. Dong, Y. X. Liu, C. P. Sun, and F. Nori, Phys. Rev. A 78, 063827 (2008).

[26] H. T. Dung and K. Ujihara, Phys. Rev. A 59, 2524 (1999).

[27] F. L. Kien, N. H. Quang, and K. Hakuta, Opt. Commun. 178, 151 (2000).

[28] As expected, Eq. (10) agrees with Eq. (8) at first order in $\Gamma t_{d}$. We stress, however, that the result in Eq. (10) is exact and thus valid for any $\Gamma t_{d}$.

[29] Q.-J. Tong, J.-H. An, H.-G. Luo, and C. H. Oh, J. Phys. B 43, 155501 (2010).

[30] This is easily seen by calculating the real space amplitude $\psi_{b}(x) \propto \int d k \varphi_{b}(k) \sin (k x)$ (see Output field dynamics). Standard contour integration methods, together with the condition $k_{0} x_{0}=n \pi$, yield $\psi_{b}(x) \propto \sin \left(k_{0} x\right) \theta\left(x_{0}-x\right)$, hence showing that the field amplitude is exactly zero for $x>x_{0}$.

[31] C. K. Law and H. J. Kimble, J. Mod. Opt. 44, 2067 (1997); A. Kuhn, M. Hennrich, T. Bondo, and G. Rempe, Appl. Phys. B 69, 373 (1999).

[32] V. Giovannetti, P. Tombesi, and D. Vitali, Phys. Rev. A 60, 1549 (1999). 